\title{
A SOLIDLY MOUNTED RESONATOR WITH CMOS-FABRICATED ACOUSTIC MIRROR FOR LOW-COST AIR QUALITY MONITORING
}

\author{
Farah H. Villa López, Marina Cole , Enrique Iborra, Mario De-Miguel Ramos and \\ Julian W. Gardner
}

\begin{abstract}
This work presents a novel Solidly Mounted Resonator (SMR) device for use in a portable, low-cost and lowpower air quality monitor. The acoustic mirror, an essential part of the SMR device for energy confinement, is fabricated within a CMOS process, and advances upon current acoustic device technology. This CMOS-fabricated acoustic mirror will enable the device to be monolithically integrated and fabricated in high volume with reduced postprocessing steps compared to the state-of-the-art acoustic based sensors. The CMOS-fabricated acoustic mirror demonstrated good reflectivity with a high quality factor of 500. Experimental results on particulate matter sensing with the CMOS based SMR sensor showed a response of $117 \mathrm{kHz} / \mathrm{ng}$ when detecting fine particles $(<1 \mu \mathrm{m})$.
\end{abstract}

\section{KEYWORDS}

Solidly mounted resonator, acoustic mirror, CMOS fabrication, SMR-IC integration, particle sensing, air quality monitoring.

\section{INTRODUCTION}

Air pollution due to airborne particulate matter, especially PM2.5 (fine particles with diameters $<2.5 \mu \mathrm{m}$ ) is considered greatly hazardous for human health. A wide range of respiratory and cardiovascular diseases such as lung cancer, asthma and heart failure have been associated to the short-term and long-term exposure to these pollutants $[1,2]$.

As society becomes aware of the adverse health effects posed by airborne particulate matter, there has been a growing demand for low-cost, low-power and portable air quality monitors. Devices such as MEMS resonators, silicon cantilevers and acoustic resonators have been proposed previously for the development of portable particle sensors [3-5].

Among the acoustic wave based devices, thin film bulk acoustic resonators (FBARs) have been recently proposed as the mass sensitive elements in a portable particle monitor due to their high sensitivity and miniature size [6]. More recently, solidly mounted resonators have received special attention because offer high sensitivity, small footprint, mechanical robustness, good power handling capabilities and the possibility for CMOS integration [7-9].

The basic structure of SMR devices consist of a thin piezoelectric layer between two metal electrodes (the resonator structure), fabricated on top of a silicon carrier substrate. To provide acoustic isolation and prevent energy leakage to the substrate, a so-called acoustic mirror, also known as Bragg reflector, is used in the SMR structure. The acoustic mirror is formed by a series of alternating layers of low and high acoustic impedance materials with typically a $\lambda / 4$ thickness.

Mass production of SMR devices (extensively used for mobile communication applications) is performed at CMOS foundries, making use of standard integrated circuity (IC) equipment [8]. However, due to the specific thicknesses required for the layers, non-standard CMOS processes are used to form these devices.

Current approaches for the integration of SMR devices with ICs require both the acoustic mirror and the resonator structure to be entirely fabricated in a post-CMOS fabrication process either above or adjacent to the integrated circuitry $[10,11]$. This post-CMOS process requires the deposition of a large number of layers and the use of several photolithography masks (typically more than 10). Thus increasing the manufacturing time and cost, reducing the manufacturing yield and decreasing the performance of the devices due to the need for external interconnections.

In this work, the development of a novel CMOS based SMR device for use in a portable air quality monitor is presented. The device advances upon the current generation of acoustic devices, through the fabrication of the acoustic mirror within a CMOS process, enabling the SMR device to be monolithically integrated and fabricated in large volumes, at low-cost and with reduced postprocessing steps.

\section{CMOS BASED SMR DEVICE}

The proposed monolithically integrated CMOS based SMR device is depicted in Figure 1.

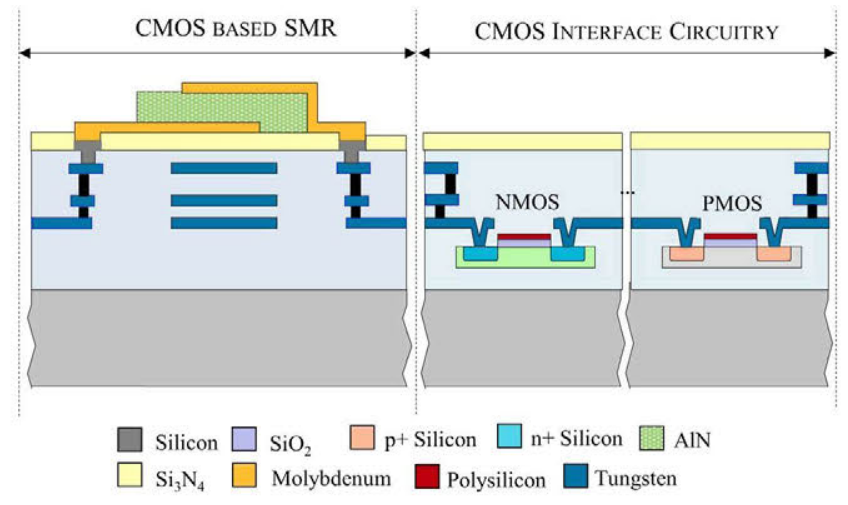

Figure. 1: Structure of the CMOS based SMR device and integration with CMOS circuitry for use within a low-cost particle sensing system. 
The CMOS based SMR device is depicted adjacent to integrated circuitry and interconnections. However, the device could also be fabricated on top of the CMOS substrate. Both the IC and the acoustic mirror are entirely fabricated during a CMOS fabrication process, whereas only the resonator structure (the piezoelectric layer and electrodes) is fabricated in a post-CMOS process.

In this work, the development of the CMOS based SMR device is presented as a proof of concept for the novel CMOS-fabricated acoustic mirror. The detailed structure of this device is depicted in Figure 2.

The high and low acoustic impedance layers of the acoustic mirror are formed during the metallization process of the selected XFAB XI10 SOI-CMOS process. The materials (tungsten and silicon dioxide) and the thicknesses of these layers are completely defined by the selected CMOS process. Only the resonator structure, formed by piezoelectric AlN and Mo electrodes, was formed in a postCMOS process.

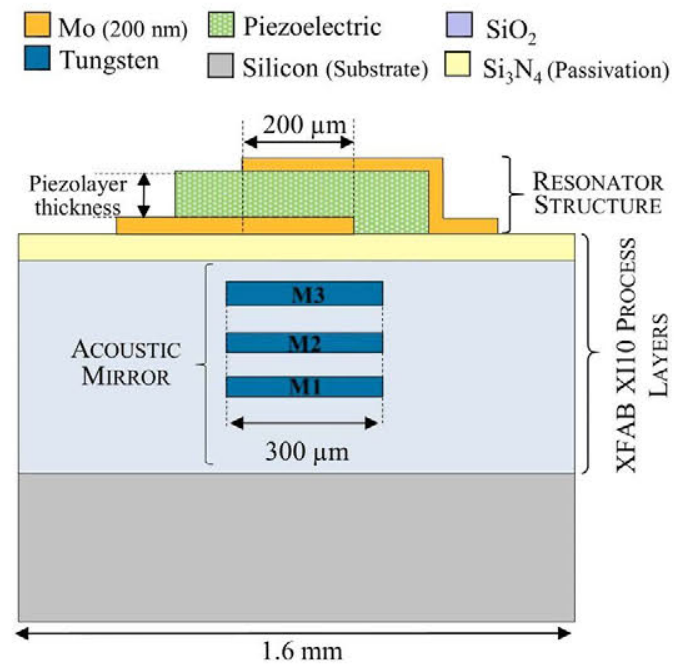

Figure. 2: Structure of the novel CMOS based SMR device. Thicknesses and materials of the acoustic mirror layers are defined by the CMOS process.

\section{Design and Modelling}

The selected XFAB XI10 CMOS process allows for the use of up to 3 layers of aluminium or tungsten metallization. Tungsten was chosen for the metallization process due to the larger acoustic impedance ratio between $\mathrm{W}$ and $\mathrm{SiO}_{2}$ (at a value of $\sim 8$ ) compared to that of $\mathrm{Al}$ and $\mathrm{SiO}_{2}$ (with a ratio of $\sim 1.3$ ).

A one-dimensional Mason model was used to simulate the mirror transmissivity prior to fabrication. For the proposed structure all three tungsten metallization layers were used to maximize the wave reflection, as shown in Figure 3. The thicknesses of the $\mathrm{W}$ and $\mathrm{SiO}_{2}$ layers to form the acoustic mirror are entirely defined by the selected XFAB XI10 CMOS process, which gives place to an asymmetric mirror structure.

Asymmetric acoustic mirrors can exhibit increased reflectivity at specific frequencies. For the proposed CMOS based acoustic mirror, excellent performance with high reflectivity and wide bandwidth was predicted at frequencies between 1.9 and $3 \mathrm{GHz}$, but also good reflectivity is observed between 3.5 and $4 \mathrm{GHz}$.

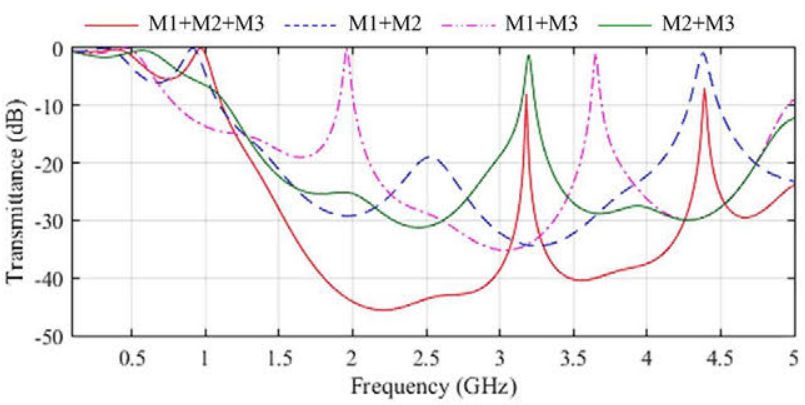

Figure. 3: Simulated transmissivity of the acoustic mirror with tungsten metallization of the XI10 process layers.

The transmissivity of the CMOS based acoustic mirror dictates the optimal operating frequency of the resonator built on top of the mirror structure. To achieve the high quality factors needed for resonance, the operating range of the resonator is bounded by the frequency range at which the mirror exhibits maximum reflectivity (desired frequencies between 1.9 and $3 \mathrm{GHz}$ ).

As part of the design process, a 2D finite element model was constructed (COMSOL Multiphysics v4.2) to verify the operation of the entire CMOS based SMR device. The model (containing all the layers in the resonator stack) was used to simulate a range of thickness of the piezoelectric layer (AIN), between $500 \mathrm{~nm}$ and $2 \mu \mathrm{m}$. The simulation results demonstrated a reasonable tolerance of layer thickness is permissible within this range for the acoustic mirror to effectively confine the energy.

\section{Fabrication and post-processing steps}

The CMOS based acoustic mirror was fabricated using the XFAB XI10 SOI-CMOS process on bulk wafers. The metal layers of the acoustic mirror were patterned with a circular shape (diameter of $300 \mu \mathrm{m}$ ). A top-view optical photograph of the CMOS fabricated acoustic mirror is shown in Figure 4a, and a cross-sectional SEM photograph of the CMOS fabricated acoustic mirror layers is shown in Figure $4 b$.

Thicknesses of the CMOS layers were estimated from the SEM photograph using an image processing software. Measured thicknesses of the metal layers were found to lay within the tolerance values given by the process specifications.

To complete the fabrication of the CMOS based SMR device, post-processing steps are required for the fabrication of the resonator structure. The post-fabrication process was performed using standard microfabrication technologies. The total number of post-processing steps was significantly reduced compared to state-of-the-art devices. These post-processing steps included the deposition of a total of three layers and the use of only one photolithography mask to pattern the top electrode.

Materials for the bottom and top electrode were deposited from high purity targets $(99.999 \%)$ by sputtering. The AlN layer was deposited on a custom-made ultra-high vacuum sputtering system (MAREA) from an Al target (99.999\% purity) in an $\operatorname{Ar} / \mathrm{N}_{2}$ mixture. The top electrode was patterned using contact photolithography and a wet etching process. 


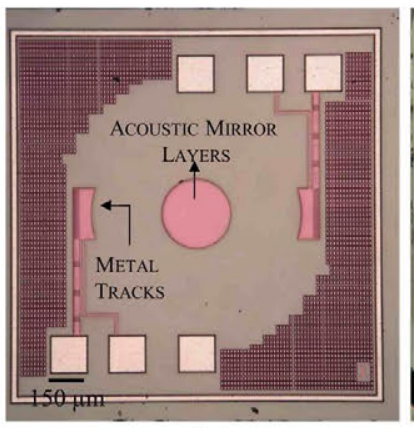

(a)

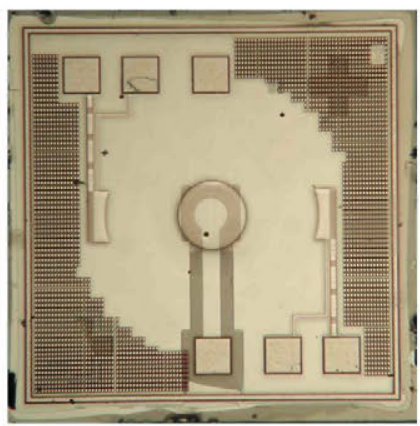

(c)

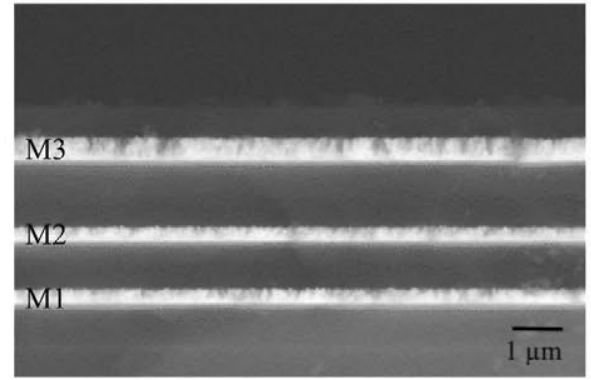

(b)

Figure 4: Fabricated devices: (a) CMOS substrate, (b) SEM photograph ofCMOS acoustic mirror layers, (c) SMR device after post-processing $(1.6 \mathrm{~mm} \times 1.6 \mathrm{~mm})$.

The post-fabrication process used a capacitive coupling method, where a large-area bottom electrode is capacitively coupled to a large metallic layer surrounding the top electrode of the SMR device. The bottom electrode is accessed through the capacitive coupling and thus no etching of the piezoelectric layer was required.

Table 1 summarizes the layer thicknesses and materials used for fabrication of the resonator structure. A photograph of the completely fabricated CMOS based SMR device after the post-processing stages is shown in Figure $4 \mathrm{c}$. The individual dies have an overall size of $1.6 \mathrm{~mm} \times 1.6 \mathrm{~mm}$.

Table 1: Summary of layers deposited during the postprocessing steps.

\begin{tabular}{|l|c|c|}
\hline Layer & Material & Thickness \\
\hline Bottom Electrode & Ti/Mo & $15 / 200 \mathrm{~nm}$ \\
\hline Piezoelectric Layer & AlN & $1800 \mathrm{~nm}$ \\
\hline Top Electrode & Mo & $150 \mathrm{~nm}$ \\
\hline
\end{tabular}

\section{EXPERIMENTAL}

Initial characterization of the fabricated CMOS based SMR devices was performed using a network analyzer in order to obtain the impedance characteristics of the device. The characterization was conducted in a one-port configuration using GSG RF probes.

Figure 5 shows the typical measured frequency response for a set of CMOS based SMR devices. As observed in the figure, these devices exhibited very similar responses and a fundamental resonant frequency of $\sim 1.9$ $\mathrm{GHz}$ was measured.

High quality factors above 500 and an effective coupling factor of $\sim 4 \%$ were measured for these devices, with an active resonator area of approximately $52,000 \mu \mathrm{m}^{2}$. The high quality factors obtained indicate that the CMOS fabricated acoustic mirror is capable of efficiently confining the acoustic energy within the resonator structure.

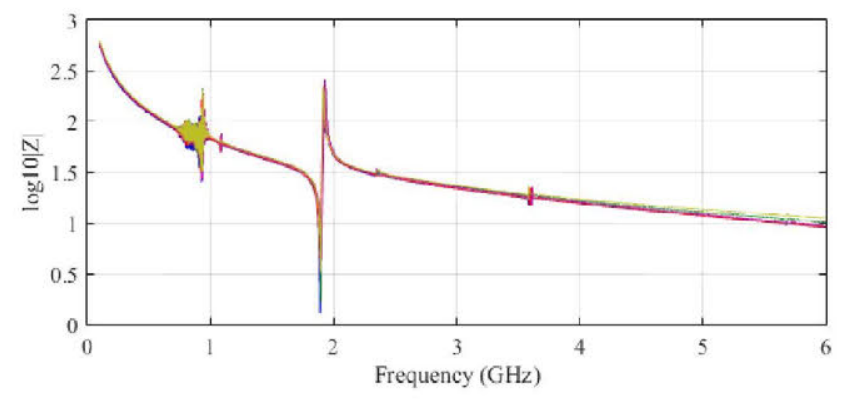

Figure 5: Measured frequency response of impedance of fabricated CMOS based SMR device.

\section{Particle Testing}

For their use in sensing applications, acoustic devices rely on a change in the properties of the propagating wave (frequency, amplitude, phase) caused by the interaction with the environment [12]. For particle sensing, the mass loading on the SMR device due to the particles deposited on the active area causes a negative shift on the resonant frequency of the device.

The CMOS based SMR device was characterized for the detection of fine PTFE particles $(<1 \mu \mathrm{m})$. Initial experiments were conducted by depositing the fine PM2.5 particles on the active area of the devices, using a micrometer wire guided by a three-axes linear stage in a similar way to that reported in [5]. Figure 6 shows the particles deposited onto the surface of the CMOS based SMR device.

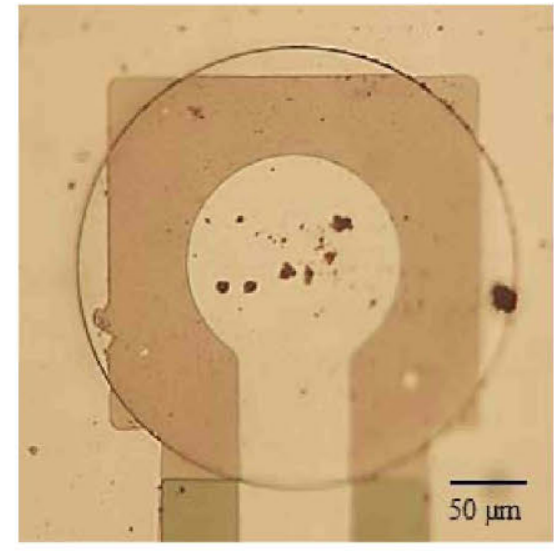

Figure 6: Photograph of the $1 \mu \mathrm{m}$ PTFE particles deposited on the sensing area of CMOS based SMR device. Particles formed clusters.

The resonant frequency of the SMR was monitored in real-time using a network analyzer, which was interfaced to a LABVIEW acquisition virtual instrument. A typical frequency response is shown in Figure 7. As the particles were deposited on the sensing area, a negative frequency shift in the $\mathrm{kHz}$ range was observed. 


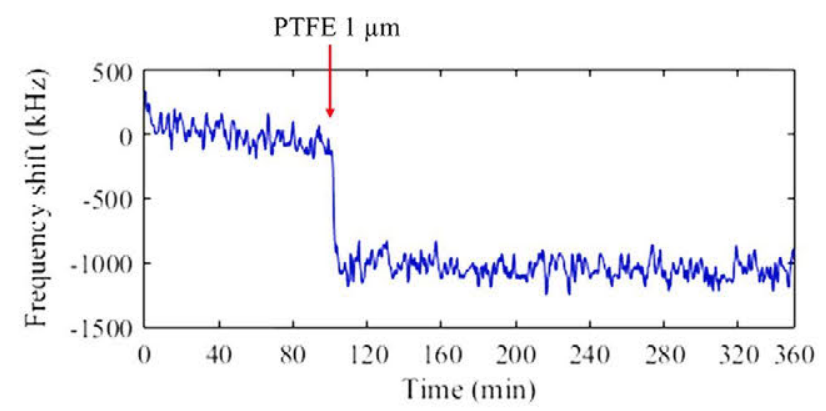

Figure 7: Measured frequency shift of the CMOS based SMR due to PTFE particle loading.

The fabricated CMOS based SMR devices were capable of detecting particle masses in the range of $1 \mathrm{ng}$ to $8.5 \mathrm{ng}$ with a mass sensitivity of $\sim 117 \mathrm{kHz} / \mathrm{ng}$. These preliminary results are shown in Figure 8 . The performance of these devices demonstrates their potential to be monolithically integrated with the IC as the main sensing element in a portable, low-cost air quality monitor.

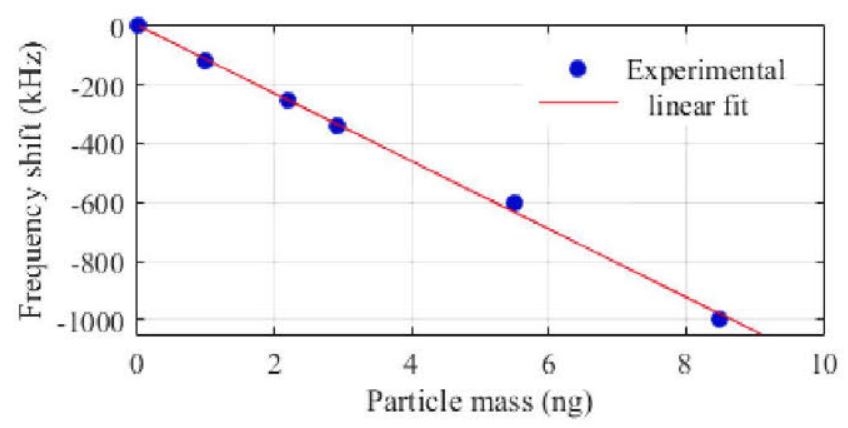

Figure 8: SMR frequency shift due to particle loading.

\section{CONCLUSIONS AND FURTHER WORK}

A novel CMOS based SMR device was designed, fabricated and characterized for use as part of smart portable particle sensor system. The device advances upon current acoustic wave device technology through the use of a CMOS fabricated acoustic mirror. The resonant frequency of the device is determined by the transmissivity of the CMOS acoustic mirror, which in turn is determined by the materials and layer thicknesses of the selected CMOS process.

The CMOS based SMR device resonates at $1.9 \mathrm{GHz}$ and exhibits a good performance with a high quality factor of 500, efficiently confining the energy within the resonator. The CMOS based SMR device was capable of detecting PTFE particles of $1 \mu \mathrm{m}$ in size with a sensitivity of $117 \mathrm{kHz} / \mathrm{ng}$.

Full characterization of the devices interfaced to driving circuitry is currently being carried out. Further work is under way towards the development of a low power, portable sensor system by fabricating this CMOS based SMR together with the integrated circuitry.

\section{ACKNOWLEDGEMENTS}

This work was funded under the EC 7th Framework Program Project No. 611887, "Multi-Sensor-Platform for Smart Building Management: MSP".

\section{REFERENCES}

[1] N. Martinelli, O. Olivieri and D. Girelli, "Air particulate matter and cardiovascular disease: A narrative review", European Journal of Internal Medicine, 24, 4 (2013), pp. 295-302.

[2] K. H. Kim, E. Kabir and S. Kabir, "A review on the human health impact of airborne particulate matter", Environment International, 74 (2015), pp. 136-143.

[3] E. Mehdizadeh, J.C. Wilson, A. Hajjam, A. Rahafrooz and S. Pourkamali, "Aerosol impactor with embedded MEMS resonant mass balance for real-time particulate mass concentration monitoring", 2013 Transducers \& Eurosensors XXVII: The International Conference on Solid-State Sensors, Actuators and Microsystems (2013), pp. 661-664.

[4] H. S. Wasisto, W. Wu, S. Merzsch, E. Uhde, A. Waag and E. Peiner, "Low-cost wearable cantilever-based nanoparticle sensor microsystem for personal health and safety monitoring", 2015 Transducers -18th International Conference on Solid-State Sensors, Actuators and Microsystems, Anchorage, Alaska (2015), pp. 428-431.

[5] S. Thomas, M. Cole, F.H. Villa-López and J.W. Gardner, "High frequency surface acoustic wave resonator-based sensor for particulate matter detection", Sensors and Actuators A: Physical, 244 (2016), pp. 138-145.

[6] J.P. Black, A. Elium, R. M. White, M. G. Apte, L. A. Gundel and R. Cambie, "MEMS-enabled miniaturized particulate matter monitor employing $1.6 \mathrm{GHz}$ aluminum nitride thin-film bulk acoustic wave resonator (FBAR) and thermophoretic precipitator", IEEE Ultrasonics Symposium. New York, NY (2007), pp. 476-479.

[7] F. Z. Bi and B.P. Barber, "Bulk acoustic wave RF technology", IEEE Microwave Magazine, 9, 5 (2008), pp. $65-80$.

[8] R. Aigner, "Volume manufacturing of BAW-filters in CMOS Fab", 2nd International Symposium on Acoustic Wave Devices for Future Mobile Communication Systems (2004), pp. 129-134.

[9] S. Thomas, F. H. Villa-López, J. Theunis, J. Peters, M. Cole and J. W. Gardner, "Particle Sensor Using Solidly Mounted Resonators", IEEE Sensors Journal (2016), 16,8 , pp. 2282-2289,

[10] K. Tukkiniemi, A. Rantala, M. Nirschl, D. Pitzer, T. Huber and M. Schreiter, "Fully integrated FBAR sensor matrix for mass detection", Procedia Chemistry, 1, 1 (2009), pp. 1051-1054.

[11]L. Elbrecht, R. Aigner, C. I. Lin and H. J. Timme, (2004). "Integration of bulk acoustic wave filters: concepts and trends", IEEE MTT-S International Microwave Symposium Digest (2004), pp. 395-398.

[12]D. S. Ballentine, R. M. White, S. J. Martin, A. J. Ricco, E. T. Zellers, G. C. Frye and H. Wohltjen, "Acoustic wave sensors: theory, design and physico-chemical applications", Academic Press, San Diego (1997). 